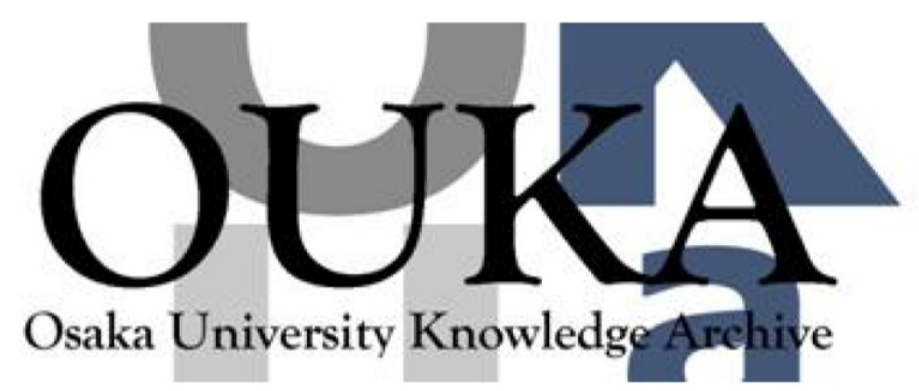

\begin{tabular}{|c|l|}
\hline Title & $\begin{array}{l}\text { Coupled Nosé-Hoover Lattice : a set of Nosé- } \\
\text { Hoover equations with different temperatures }\end{array}$ \\
\hline Author(s) & Fukuda, Ikuo \\
\hline Citation & Physics Letters A. 380(33) p. 2465-p. 2474 \\
\hline Issue Date & \\
\hline oaire:version & AM \\
\hline URL & https://hdl. handle. net/11094/54591 \\
\hline rights & \\
\hline Note & \\
\hline
\end{tabular}

Osaka University Knowledge Archive : OUKA

https://ir. Library. osaka-u. ac. jp/

Osaka University 


\title{
Coupled Nosé-Hoover Lattice: a set of Nosé-Hoover equations with different temperatures
}

\author{
Ikuo Fukuda \\ Institute for Protein Research, Osaka University, Osaka 565-0871, Japan
}

\begin{abstract}
A simple scheme was presented to couple any number of the Nosé-Hoover equations with different heat-bath temperatures. In general, several practical procedures can be considered to realize such a coupling, where the system is under nonequilibrium. However, the present scheme provides an equilibrium distribution, namely, a smooth invariant measure. This is attained by a very simple idea, that is, a force scaling. The current scheme realizes the coupled differential equations, analogous to the coupled maps. Theoretical possibilities, mathematical framework, and practical utilities are discussed. Numerical validations applying the method to a simple two-oscillator system are given.
\end{abstract}

Key words: Nosé-Hoover method, molecular dynamics, continuous dynamical system

\section{Introduction}

Thirty years ago, Nosé [1] formulated a constant temperature molecular dynamics (MD) scheme that is compatible with the canonical distribution. This is based on the Newtonian equations of motion (EOM), represented by the coordinates and the corresponding momenta of a given physical system, and is made by attaching an additional degree of freedom describing a heat bath at an arbitrary fixed temperature. He defines the Hamiltonian represented by these coordinates, momenta, and the additional variables, and then propose a (non-symplectic) variable transformation and a time transformation to obtain EOMs physically sound. The underling essential physical feature was then revealed by Hoover [2], eliminating a redundant variable and creating a perspicaciously defined variable. This newly defined variable represents a dynamical friction coefficient, which plays a role of thermostat for the physical system. The obtained EOMs are called the Nosé-Hoover (NH) equations. 
Many MD simulations have been performed using the NH equations and they have revealed characteristics of physical systems in terms of microscopic descriptions [3-5].

The NH equations describes the equilibrium characterized by the canonical distribution at the fixed one temperature. If we add two, or more, thermostat variables, however, then we are allowed to simulate a system under a nonequilibrium, by defining distinct temperatures for the plural heat bathes that are described by the appended thermostat variables. Two bathes, hot and cold, are used to study heat flow [6,7]. Such scheme should also be useful to handle Born-Oppenheimer assumption by keeping nuclear degrees of freedom hot and electronic degrees of freedom cold. It could also be efficient to investigate more complicated system, such as biomolecular system, where only solute molecules can be destabilized by hot bath so that the enhanced sampling would be attained. However, the bottle neck for wider applications is that these $\mathrm{NH}$ systems attached with plural heat bath degree of freedom are not under equilibrium and yield no statistical descriptions. Thus it is difficult to obtain thermodynamical quantities, including free energy.

This work presents a simple scheme that any number of the NH equations are coupled and the obtained total system allows a complete statistical description. The heat baths of the individual $\mathrm{NH}$ equations have different temperatures in general. On the contrary, the total system has a natural distribution density function, which yields the statistical description. Surprisingly, these two contradictory issues can be harmonized by a very simple manner.

Two gains would be expected. First, the presented new ordinary differential equation (ODE) should be interesting from a viewpoint of continuous dynamical system. Here, note that the coupled map lattices [8,9] couple plural chaotic maps (each map defines a discrete-time development of a system; note the system is often defined in an abstract sense). The manner of the coupling of these maps is based on a kind of averaging or assembling the contributions of individual maps. The coupled maps show very interesting behavior, including chaotic itinerancy, hierarchical dynamics and clustering. They have provided new ideas and close relationship to many systems that appear in biology, complex systems, and brain science. The current scheme presents a method to couple plural chaotic (or no chaotic) ODEs (each ODE defines a continuous-time development of a system). The manner of the coupling of these NH ODEs is based on setting a general potential function that would assemble the contributions of individual NH ODE systems. On the basis of an expectation to the feature provided by these ideas, this paper emphasizes the aspect of this first issue. We call the current method by coupled Nosé-Hoover $(\mathrm{NH})$ lattice.

The second issue we expect the gain is in the feature that the total system 
has an equilibrium distribution (smooth invariant measure). It may be interesting in the fundamental aspect of physical science. It would also be efficient in a more practical purpose such as effective sampling of complicated physical systems, as described above. Furthermore, other recipe for the sampling may be provided. Consider a target physical system whose effective sampling of states under the room temperature is sought. Then also consider many systems that are copies of the target system and define the interactions between these copied systems and the original system. These individual copied systems can be subjected to have higher/lower temperatures in an arbitrary manner. The interaction between the original system and higher temperature systems may provide a sufficiently high energy to surmount an energy barrier for efficiently exploring the original system's phase space. The interaction between the original system and lower temperature systems may help to sample low energy states. (These gains are similar to that of the replica exchange MD method [10] in that the interactions should be regarded as the exchange of the states or temperatures.) The point is that we can have the required information of the target system under the canonical distribution by using a reweighting procedure, because the distribution density of the total system is given in advance. Furthermore, in fact, these systems are not necessarily the copy of the original target system, but can be any systems as long as the interactions between the systems can be suitably defined.

In Sec. II, the Nosé-Hoover equation with one degree of freedom is reviewed. In Sec. III, coupling scheme for the Nosé-Hoover equations where each equation has one degree of freedom is proposed for simplicity and analyzed in detail. In Sec. IV, coupling scheme for a more general case is presented and a reweighting method is discussed to assess the state sampling. Sec. V address numerical simulation results for a model system composed from oscillators. We confirm that the current scheme provides interesting dynamical features and yields satisfactory results.

\section{Review of the Nosé-Hoover equation}

The NH equation with one degree of freedom is given by

$$
\left.\begin{array}{l}
\dot{x}=p \\
\dot{p}=-D U(x)-(\zeta / \mathrm{Q}) p, \\
\dot{\zeta}=p^{2}-T
\end{array}\right\}
$$

where $x \in \mathbb{R}^{1}$ is the coordinate for the physical system with one degree of freedom, $p \in \mathbb{R}^{1}$ is the corresponding momenta, and $U(x)$ is the potential energy. We put a unit mass for the particle for simplicity. $\zeta \in \mathbb{R}$ is a variable 
to control the temperature of the original physical system and to set it to a given value of the external temperature $T$. Q is Nosé's mass, a positive parameter. This ODE has an invariant density, or equilibrium distribution density, $\rho(\omega)=\rho(x, p, \zeta)$, defined by

$$
\rho(x, p, \zeta)=\exp \left[-\frac{1}{T}\left(U(x)+\frac{1}{2} p^{2}+\frac{1}{2 \mathrm{Q}} \zeta^{2}\right)\right] .
$$

This can be confirmed by the validity of the equilibrium Liouville equation

$$
\operatorname{div} \rho X=0,
$$

where $X(\omega)=X(x, p, \zeta)$ is defined by the RHS of Eq. (1). For an ergodic assumption, for any phase-space function $g$, we have

$$
\bar{g}=\langle g\rangle .
$$

Here $\bar{g}$ is the long time average

$$
\bar{g} \equiv \lim _{\tau \rightarrow \infty} \frac{1}{\tau} \int_{0}^{\tau} g(x(t), p(t), \zeta(t)) d t
$$

and $\langle g\rangle$ is the space average

$$
\langle g\rangle \equiv \int_{\mathbb{R}^{3}} g(\omega) \rho(\omega) d \omega / \int_{\mathbb{R}^{3}} \rho(\omega) d \omega .
$$

Thus, for a physical system variables $(x, p)$ we have the canonical distribution $\exp \left[-\frac{1}{T}\left(U(x)+\frac{1}{2} p^{2}\right)\right] d x d p$ at the temperature $T$.

\section{Coupled Nosé-Hoover lattice}

We will couple $N$ NH equations, each of which is based on Eq. (1). Quantities originated from each $\mathrm{NH}$ equation will be indicated by attaching superscript $[I]$ or $[J]$ that runs from 1 to $N$. Thus we have $N$ triplets, $\left(x^{[I]}, p^{[I]}, \zeta^{[I]}\right)$, $I=1, \ldots, N$, to define a total space that is the $N$ product of $\mathbb{R}^{3}$. By the coupling of $N$ NH systems, we want to define the "interaction" of these systems via the potential function, such as $U\left(x^{[1]}, x^{[2]}, \ldots, x^{[N]}\right)[U$ is now a function of $N$ variables]. In addition, we are interested in a situation that each system has different heat bath; viz., distinct temperature $T^{[I]}$ and Nosé's mass $\mathrm{Q}^{[I]}$, $I=1, \ldots, N$. The proposed EOMs for this total space is:

$$
\left.\begin{array}{l}
\dot{x}^{[I]}=p^{[I]}, I=1, \ldots, N \\
\dot{p}^{[I]}=-\nu T^{[I]} D_{I} U(x)-\left(\zeta^{[I]} / \mathrm{Q}^{[I]}\right) p^{[I]}, I=1, \ldots, N, \\
\dot{\zeta}^{[I]}=\left(p^{[I]}\right)^{2}-T^{[I]}, I=1, \ldots, N .
\end{array}\right\}
$$


Here $D_{I} U(x)=D_{I} U\left(x^{[1]}, x^{[2]}, \ldots, x^{[N]}\right)$ indicates a partial derivative of $U$ with respect to the $I$ th variable at product-space coordinate $x=\left(x^{[1]}, x^{[2]}, \ldots, x^{[N]}\right) \in$ $\mathbb{R}^{N}$. Thus $-D_{I} U(x)$ is the force acting to the $I$ th system in this total productspace. The factor, $\nu T^{[I]}$, attached to this force may seem to be peculiar. This factor is introduced to satisfy the Liouville equation in this new system, as seen below. $\nu$ is a parameter that should be suitably taken. Now we define a density of the total space as follows:

$$
\begin{aligned}
\rho(x, p, \zeta) & \equiv \rho\left(x^{[1]}, \ldots, x^{[N]}, p^{[1]}, \ldots, p^{[N]}, \zeta^{[1]}, \ldots, \zeta^{[N]}\right) \\
& =\prod_{I=1}^{N} \exp \left[-\frac{1}{T^{[I]}}\left(\mu U(x)+\frac{1}{2}\left(p^{[I]}\right)^{2}+\frac{1}{2 Q^{[I]}}\left(\zeta^{[I]}\right)^{2}\right)\right] .
\end{aligned}
$$

Here $\mu$ is a parameter that are related to the parameter $\nu$ such that

$$
\nu=\left(\sum_{J=1}^{N} \frac{1}{T^{[J]}}\right) \mu .
$$

Now we prove that the Liouville equation

$$
\operatorname{div} \rho X=0
$$

holds, where $\rho$ is given in Eq. (8) and $X(\omega) \equiv X(x, p, \zeta) \equiv$ $X\left(x^{[1]}, \ldots, x^{[N]}, p^{[1]}, \ldots, p^{[N]}, \zeta^{[1]}, \ldots, \zeta^{[N]}\right)$ is defined by the RHS of Eq. (7). Notice $\operatorname{div} \rho X(\omega)=(\nabla \rho(\omega) \mid X(\omega))+\rho(\omega) \operatorname{div} X(\omega)=\sum_{a=1}^{3 N} D_{a} \rho(\omega) X_{a}(\omega)+$ $\rho(\omega) \sum_{a=1}^{3 N} D_{a} X_{a}(\omega)$ and since $\rho(\omega)>0$ for any point $\omega$, where our notation is $\omega \equiv(x, p, \zeta) \equiv\left(x^{[1]}, \ldots, x^{[N]}, p^{[1]}, \ldots, p^{[N]}, \zeta^{[1]}, \ldots, \zeta^{[N]}\right)$ $\equiv\left(\omega_{1}, \ldots, \omega_{3 N}\right) \in \mathbb{R}^{3 N}$. Thus Eq. (10) is equivalent to

$$
(\nabla \ln \rho(\omega) \mid X(\omega))+\operatorname{div} X(\omega)=0
$$

for any $\omega \in \mathbb{R}^{3 N}$. Now, from the aid of Eq. (9), we see

$$
\begin{aligned}
\ln \rho(x, p, \zeta) & =-\sum_{J=1}^{N} \frac{1}{T^{[J]}}\left(\mu U(x)+\frac{1}{2}\left(p^{[J]}\right)^{2}+\frac{1}{2 \mathrm{Q}^{[J]}}\left(\zeta^{[J]}\right)^{2}\right) \\
& =-\nu U(x)-\sum_{J=1}^{N} \frac{1}{T^{[J]}}\left(\frac{1}{2}\left(p^{[J]}\right)^{2}+\frac{1}{2 \mathrm{Q}^{[J]}}\left(\zeta^{[J]}\right)^{2}\right)
\end{aligned}
$$

Thus we easily get

$$
\begin{aligned}
& D_{x^{[I]}} \ln \rho(\omega)=-\nu D_{I} U(x), I=1, \ldots, N, \\
& D_{p^{[I]} \ln \rho(\omega)}=-\frac{1}{T^{[I]}} p^{[I]}, I=1, \ldots, N, \\
& D_{\zeta^{[I]} \ln \rho(\omega)}=-\frac{1}{T^{[I]}} \frac{\zeta^{[I]}}{\mathrm{Q}^{[I]}}, I=1, \ldots, N .
\end{aligned}
$$


On the other hand, the divergence for Eq. (7) becomes the sum of the divergence for the original NH equation, viz.,

$$
\operatorname{div} X(\omega)=-\sum_{I=1}^{N} \frac{\zeta^{[I]}}{\mathrm{Q}^{[I]}} .
$$

Integrating these results, we have

$$
\begin{aligned}
& (\nabla \ln \rho(\omega) \mid X(\omega))+\operatorname{div} X(\omega) \\
& =-\nu \sum_{I=1}^{N} D_{I} U(x) p^{[I]} \\
& +\sum_{I=1}^{N} \frac{1}{T^{[I]}} p^{[I]}\left[\nu T^{[I]} D_{I} U(x)+\left(\zeta^{[I]} / \mathrm{Q}^{[I]}\right) p^{[I]}\right] \\
& -\sum_{I=1}^{N} \frac{1}{T^{[I]}} \frac{\zeta^{[I]}}{\mathrm{Q}^{[I]}}\left[\left(p^{[I]}\right)^{2}-T^{[I]}\right] \\
& -\sum_{I=1}^{N} \frac{\zeta^{[I]}}{\mathrm{Q}^{[I]}} \\
& =0
\end{aligned}
$$

for any $\omega \in \mathbb{R}^{3 N}$. Therefore we confirm the validity of the Liouville equation, Eq. (10).

The Liouville equation ensures that $\rho$ becomes an invariant density ( $\rho d \omega$ is an invariant measure) of the flow generated by the ODE, $\dot{\omega}=X(\omega)$ [Eq. (7)] [11,12]. Therefore, the long time average for any phase space map $g: \mathbb{R}^{3 N} \rightarrow \mathbb{R}$ exists (for almost everywhere) from Birkhoff's ergodic theorem, and by assuming the ergodic condition, we have the same type equality as Eq. (4), viz.,

$$
\bar{g}=\langle g\rangle .
$$

Here $\bar{g}$ is the long time average

$$
\begin{aligned}
\bar{g} & \equiv \lim _{\tau \rightarrow \infty} \frac{1}{\tau} \int_{0}^{\tau} g(x(t), p(t), \zeta(t)) d t \\
& =\lim _{\tau \rightarrow \infty} \frac{1}{\tau} \int_{0}^{\tau} g\left(x^{[1]}(t), \ldots, x^{[N]}(t), p^{[1]}(t), \ldots, p^{[N]}(t), \zeta^{[1]}(t), \ldots, \zeta^{[N]}(t)\right) d t
\end{aligned}
$$

and $\langle g\rangle$ is the space average

$$
\begin{aligned}
\langle g\rangle & \equiv \int_{\mathbb{R}^{3 N}} g \rho d \omega / \int_{\mathbb{R}^{3 N}} \rho d \omega \\
& =\frac{\int_{\mathbb{R}^{3 N}} d x d p d \zeta g(x, p, \zeta) \prod_{I=1}^{N} \exp \left[-\frac{1}{T^{[I]}}\left(\mu U(x)+\frac{1}{2}\left(p^{[I]}\right)^{2}+\frac{1}{2 \mathrm{Q}^{[I]}}\left(\zeta^{[I]}\right)^{2}\right)\right]}{\int_{\mathbb{R}^{3 N}} d x d p d \zeta \prod_{I=1}^{N} \exp \left[-\frac{1}{T^{[I]}}\left(\mu U(x)+\frac{1}{2}\left(p^{[I]}\right)^{2}+\frac{1}{2 \mathrm{Q}^{[I]}}\left(\zeta^{[I]}\right)^{2}\right)\right]} .
\end{aligned}
$$


We have observed that the factor, $\nu T^{[I]}$, of the force and the relationship (9), between $\mu$ and $\nu$, are necessary to valid the Liouville equation. A natural choice of $\mu$ is

$$
\mu=\frac{1}{N}
$$

In this case Eq. (9) yields

$$
\begin{aligned}
\nu & =\left(\sum_{J=1}^{N} \frac{1}{T^{[J]}}\right) \mu \equiv\left(\sum_{J=1}^{N} \beta^{[J]}\right) \mu \\
& =\frac{1}{N}\left(\sum_{J=1}^{N} \beta^{[J]}\right) \equiv\langle\beta\rangle .
\end{aligned}
$$

Namely, $\nu$ becomes the average of the inverse temperatures, $\beta^{[1]}, \ldots, \beta^{[N]}$. So, the factorized force becomes,

$$
\begin{aligned}
& -\nu T^{[I]} D_{I} U(x) \\
& =-\frac{\langle\beta\rangle}{\beta^{[I]}} D_{I} U(x) \\
& \simeq-D_{I} U(x),
\end{aligned}
$$

viz., the ordinary one, if $\beta^{[I]} \simeq\langle\beta\rangle$. In other words, the factor becomes large for a smaller $\beta^{[I]}$ (or a higher temperature $T^{[I]}$ ). The density, Eq. (8), becomes [see Eq. (12)]

$$
\begin{aligned}
\rho(x, p, \zeta) & =\exp \left[-\nu U(x)-\sum_{J=1}^{N} \frac{1}{T^{[J]}}\left(\frac{1}{2}\left(p^{[J]}\right)^{2}+\frac{1}{2 \mathrm{Q}^{[J]}}\left(\zeta^{[J]}\right)^{2}\right)\right] \\
& =\exp [-\langle\beta\rangle U(x)] \prod_{I=1}^{N} \exp \left[-\beta^{[I]}\left(\frac{1}{2}\left(p^{[I]}\right)^{2}+\frac{1}{2 \mathrm{Q}^{[I]}}\left(\zeta^{[I]}\right)^{2}\right)\right]
\end{aligned}
$$

Namely, for the product-space coordinates $x=\left(x^{[1]}, \ldots, x^{[N]}\right) \in \mathbb{R}^{N}$, we have the canonical form with the average inverse temperature, $\exp [-\langle\beta\rangle U(x)]$, and for the product-space momenta $p=\left(p^{[1]}, \ldots, p^{[N]}\right) \in \mathbb{R}^{N}$ and for control variables $\zeta=\left(\zeta^{[1]}, \ldots, \zeta^{[N]}\right) \in \mathbb{R}^{N}$ we have the simple product of the individual density $\exp \left[-\beta^{[I]}\left(\frac{1}{2}\left(p^{[I]}\right)^{2}+\frac{1}{2 \mathrm{Q}^{[I]}}\left(\zeta^{[I]}\right)^{2}\right)\right]$. In practical application, uniform Nosé's masses, viz., $\mathrm{Q}^{[1]}=\cdots=\mathrm{Q}^{[N]}$, would be sufficient.

To illustrate the scheme, we consider the most basic system, a coupled system of two 1-dimensional harmonic oscillator $(1 \mathrm{HO}) \mathrm{NH}$ systems $(N=2)$; see Fig. 1. Namely, Eq. (7) with potential function

$$
U\left(x^{[1]}, x^{[2]}\right)=\frac{1}{2} \sum_{I, J=1}^{2} k^{[I, J]} x^{[I]} x^{[J]},
$$

where $k^{[I, J]}$ is the spring constant $\left(k^{[2,1]}=k^{[1,2]}\right)$ is used. The EOMs are 
explicitly written as

$$
\left\{\begin{array}{l}
\dot{x}^{[1]}=p^{[1]} \\
\dot{p}^{[1]}=-\nu T^{[1]}\left(k^{[1,1]} x^{[1]}+k^{[1,2]} x^{[2]}\right)-\left(\zeta^{[1]} / \mathrm{Q}^{[1]}\right) p^{[1]}, \\
\dot{\zeta}^{[1]}=\left(p^{[1]}\right)^{2}-T^{[I]}, \\
\dot{x}^{[2]}=p^{[2]} \\
\dot{p}^{[2]}=-\nu T^{[2]}\left(k^{[2,2]} x^{[2]}+k^{[2,1]} x^{[1]}\right)-\left(\zeta^{[2]} / \mathrm{Q}^{[2]}\right) p^{[2]}, \\
\dot{\zeta}^{[2]}=\left(p^{[2]}\right)^{2}-T^{[2]} .
\end{array}\right\}
$$

Let $T^{[1]}<T^{[2]}$ : the oscillator in system 1 is subject to a cold bath and the oscillator in system 2 a hot bath. If the coupling spring constant $k^{[1,2]}(=$ $\left.k^{[1,2]}\right)$ is zero, then the two systems are decoupled and just two $1 \mathrm{HO} \mathrm{NH}$ systems are obtained (the change $k^{[I, I]} \rightarrow T^{[I]} k^{[I, I]}$ is just the spring constant change). If they are not zeros, then the two systems couple and the coupling force is $\nu T^{[1]} k^{[1,2]} x^{[2]}$ for system 1 and $\nu T^{[2]} k^{[2,1]} x^{[1]}$ for system 2 . It should be noticed that the coupling strengths $\nu T^{[1]} k^{[1,2]}$ and $\nu T^{[2]} k^{[2,1]}$ are not equal because $T^{[1]}<T^{[2]}$. This symmetry breaking is a key of the trick to produce an equilibrium in the current method.

\section{Coupled Nosé-Hoover lattice: a general case}

In the previous section we have the coupling of the $\mathrm{NH}$ equations based on Eq. (1) that is for one degree of freedom. In general, we should work with the system with any numbers of degrees of freedom in an application. Thus we seek the form of the coupling of the $\mathrm{NH}$ equations based on $n$ degrees of freedom:

$$
\left.\begin{array}{l}
\dot{x}=\mathbf{M}^{-1} p \in \mathbb{R}^{n}, \\
\dot{p}=-\nabla U(x)-(\zeta / \mathrm{Q}) p \in \mathbb{R}^{n}, \\
\dot{\zeta}=2 K(p)-n k_{\mathrm{B}} T \in \mathbb{R}^{1},
\end{array}\right\}
$$

where $x \equiv\left(x_{1}, \ldots, x_{n}\right) \in \mathbb{R}^{n}$ are the coordinates of a certain physical system and $p \equiv\left(p_{1}, \ldots, p_{n}\right) \in \mathbb{R}^{n}$ are the corresponding momenta. $K(p)=$ $\left(p \mid \mathbf{M}^{-1} p\right) / 2 \equiv \sum_{i, j=1}^{n}\left(\mathbf{M}^{-1}\right)_{i j} p_{i} p_{j} / 2$ is the kinetic energy, with $\mathbf{M}$ being the masses (a symmetric, positive-definite square matrix of size $n$ ) of the particles, and $k_{\mathrm{B}}$ is Boltzmann's constant.

Here, we couple $N$ NH systems, each of which has $n_{I}$ degrees of freedom for $I=1, \ldots, N$. The total space of this coupling will thus be $\mathbb{R}^{\sum_{I=1}^{N}\left(2 n_{I}+1\right)}$, and 
the point $\omega$ will be represented as

$$
\omega \equiv(x, p, \zeta) \equiv\left(x^{[1]}, \ldots, x^{[N]}, p^{[1]}, \ldots, p^{[N]}, \zeta^{[1]}, \ldots, \zeta^{[N]}\right) \in \Omega \equiv \mathbb{R}^{\sum_{I=1}^{N}\left(2 n_{I}+1\right)}
$$

with coordinates, momenta, and the friction variable being

$$
\begin{aligned}
& x^{[I]}=\left(x_{1}^{[I]}, \ldots, x_{n_{I}}^{[I]}\right) \in \mathbb{R}^{n_{I}}, I=1, \ldots, N, \\
& p^{[I]}=\left(p_{1}^{[I]}, \ldots, p_{n_{I}}^{[I]}\right) \in \mathbb{R}^{n_{I}}, I=1, \ldots, N, \\
& \zeta^{[I]} \in \mathbb{R}^{1}, I=1, \ldots, N,
\end{aligned}
$$

respectively. Then, the EOM, $\dot{\omega}=X(\omega)$, that generalizes Eq. (7) to handle any degrees of freedom case is given as follows:

$$
\left\{\begin{array}{l}
\dot{x}^{[I]}=\mathbf{M}^{[I]-1} p^{[I]} \in \mathbb{R}^{n_{I}}, I=1, \ldots, N, \\
\dot{p}^{[I]}=-\nu T^{[I]} \nabla_{I} U(x)-\left(\zeta^{[I]} / \mathrm{Q}^{[I]}\right) p^{[I]} \in \mathbb{R}^{n_{I}}, I=1, \ldots, N, \\
\dot{\zeta}^{[I]}=2 K^{[I]}\left(p^{[I]}\right)-n_{I} k_{\mathrm{B}} T^{[I]} \in \mathbb{R}^{1}, I=1, \ldots, N,
\end{array}\right.
$$

or explicitly,

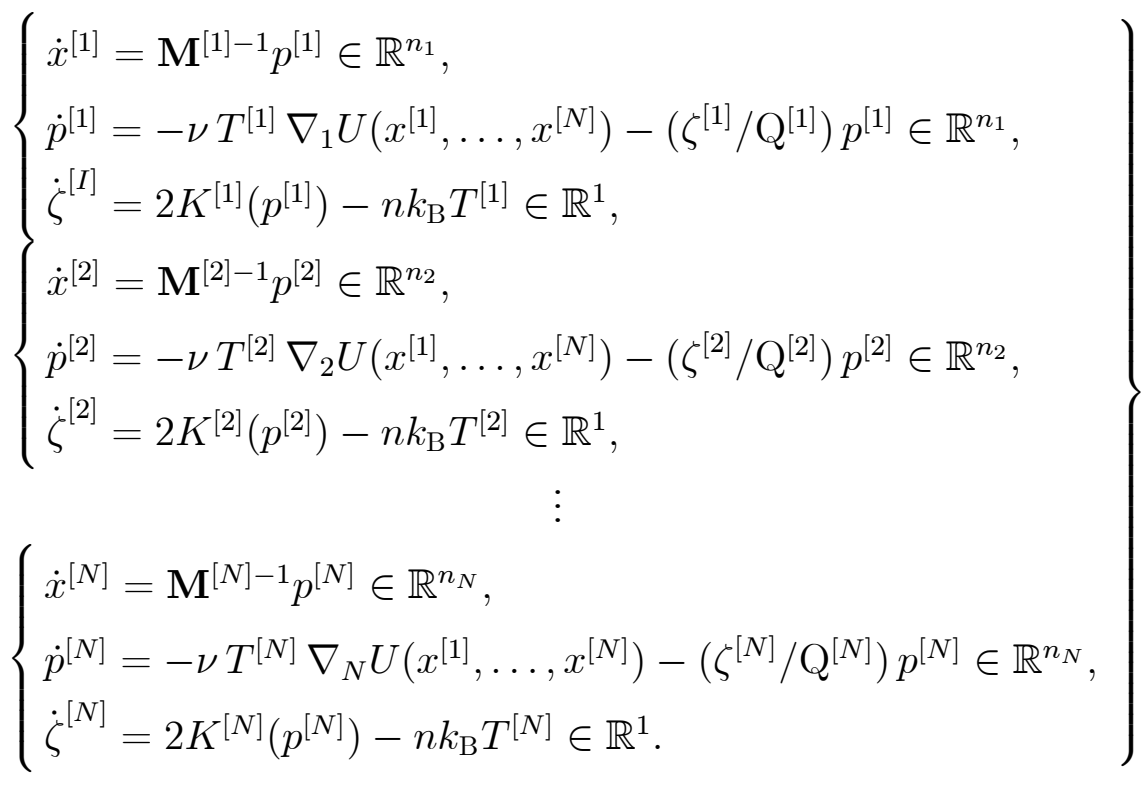

We call this a coupled NH lattice equation. Here $K^{[I]}\left(p^{[I]}\right)=\left(p^{[I]} \mid \mathbf{M}^{[I]-1} p^{[I]}\right) / 2 \equiv$ $\sum_{i, j=1}^{n_{I}}\left(\mathbf{M}^{[I]-1}\right)_{i j} p_{i}^{[I]} p_{j}^{[I]} / 2, T^{[I]}$, and $\mathrm{Q}^{[I]}$ are the kinetic energy, the temperature, and the Nosé's mass of the $I$ th system, respectively. We define a density of 
this system:

$$
\begin{aligned}
\rho(x, p, \zeta) & \equiv \rho\left(x^{[1]}, \ldots, x^{[N]}, p^{[1]}, \ldots, p^{[N]}, \zeta^{[1]}, \ldots, \zeta^{[N]}\right) \\
& =\prod_{I=1}^{N} \exp \left[-\beta^{[I]}\left(\mu U\left(x^{[1]}, \ldots, x^{[N]}\right)+K^{[I]}\left(p^{[I]}\right)+\frac{1}{2 \mathrm{Q}^{[I]}}\left(\zeta^{[I]}\right)^{2}\right)\right]
\end{aligned}
$$

where $\beta^{[I]}=1 / k_{\mathrm{B}} T^{[I]}$ for each $I$. We also define a relationship, which is essentially the same as Eq. (9), as follows:

$$
\nu=\left(\sum_{J=1}^{N} \beta^{[J]}\right) \mu .
$$

Thus

$\rho(x, p, \zeta)=\exp \left[-\nu U\left(x^{[1]}, \ldots, x^{[N]}\right)\right] \prod_{I=1}^{N} \exp \left[-\beta^{[I]}\left(K^{[I]}\left(p^{[I]}\right)+\frac{1}{2 \mathrm{Q}^{[I]}}\left(\zeta^{[I]}\right)^{2}\right)\right]$.

Note that the choice of Eq. (19) yields $\nu=\frac{1}{N}\left(\sum_{J=1}^{N} \beta^{[J]}\right)=\langle\beta\rangle$, which is the same as Eq. (20). All the results of the preceding section, including the EOMs and the corresponding density, can be obtained if we put $k_{\mathrm{B}}=1$ and $n_{I}=1$ and $\mathbf{M}^{[I]}=1(I=1, \ldots, N)$.

Now, also in this general case, we see that the Liouville equation

$$
\operatorname{div} \rho X=0
$$

holds, as a similar manner done in the previous section. Thus, under an ergodic assumption, we have

$$
\bar{g}=\langle g\rangle
$$

for map $g: \Omega \rightarrow \mathbb{R}$. Here $\bar{g}$ is the long time average

$$
\begin{aligned}
\bar{g} & \equiv \lim _{\tau \rightarrow \infty} \frac{1}{\tau} \int_{0}^{\tau} g(x(t), p(t), \zeta(t)) d t \\
& =\lim _{\tau \rightarrow \infty} \frac{1}{\tau} \int_{0}^{\tau} g\left(x^{[1]}(t), \ldots, x^{[N]}(t), p^{[1]}(t), \ldots, p^{[N]}(t), \zeta^{[1]}(t), \ldots, \zeta^{[N]}(t)\right) d t
\end{aligned}
$$

and $\langle g\rangle$ is the space average

$$
\begin{aligned}
\langle g\rangle & \equiv \int_{\Omega} g \rho d \omega / \int_{\Omega} \rho d \omega \\
& =\frac{\int_{\Omega} d x d p d \zeta g(x, p, \zeta) \rho_{\mathrm{X}}(x) \rho_{2}(p, \zeta)}{\int_{\Omega} d x d p d \zeta \rho_{\mathrm{X}}(x) \rho_{2}(p, \zeta)} .
\end{aligned}
$$


Here we have defined

$$
\begin{aligned}
\rho_{\mathrm{X}}(x) & \equiv \prod_{I=1}^{N} \exp \left[-\beta^{[I]}\left(\mu U\left(x^{[1]}, \ldots, x^{[N]}\right)\right)\right] \\
& =\exp \left[-\nu U\left(x^{[1]}, \ldots, x^{[N]}\right)\right]
\end{aligned}
$$

viz., the coordinates part of the density,

$$
\rho_{2}(p, \zeta) \equiv \prod_{I=1}^{N} \exp \left[-\beta^{[I]}\left(K^{[I]}\left(p^{[I]}\right)+\frac{1}{2 \mathrm{Q}^{[I]}}\left(\zeta^{[I]}\right)^{2}\right)\right]
$$

viz., the momenta- $\zeta$ part of the density, and $d x=d x^{[1]} d x^{[2]} \cdots d x^{[N]}=$ $d x_{1}^{[1]} \cdots d x_{n_{1}}^{[1]} \cdots d x_{1}^{[N]} \cdots d x_{n_{N}}^{[N]}$, etc. By this formalism we can set "interactions" of any type systems with different number of degrees of freedom, through $U\left(x^{[1]}, \ldots, x^{[N]}\right)$. This advantage would be clear in many applications, including multi-scale physics simulation.

The distribution of system $I$ is given by the marginal distribution density,

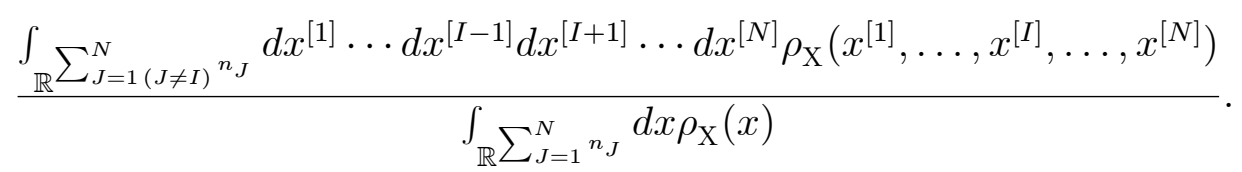

This integration is not explicitly done in general, except simple cases. However, we can obtain the information about the states in system $I$ under any distribution by using a reweighting method. Thus the impossibility of the explicit integration would not be a serious drawback of the current method. The details are discussed in the following subsection.

Remark 1: The coordinate part of the density [Eq. (37)] $\rho_{\mathrm{X}}=\exp [-\nu U]$ : $\mathbb{R}^{\sum_{J=1}^{N} n_{J}} \rightarrow \mathbb{R}$ should be smooth and integrable. The EOM (28) can be defined without this integrability condition, such as the case in which a set of $\mathrm{NH}$ equations of the ideal gases is treated. However, the integration in Eq. (36) diverges and yields meaningless result to ensure the statistical statement.

Remark 2: Equation (29) seems to be similar to the massive NH equations [13]. In fact, the essential difference between this method and the current method is the presence of the factor $\nu T^{[I]}$ in the current method. The former method has no equilibrium density, Eq. (32), and could not have any density, if individual heat baths have different temperatures, in general. Regarding the use of the $\mathrm{NH}$ thermostat in the current method, the extension of the current method based on other thermostat [14] is not difficult. For example, replacing the $\mathrm{NH}$ to the NH chains [15] is straightforward. Application to the HooverHolian method [16] should be interesting, where a strong mixing-like behavior is expected. The current method is different from the work in Refs. [17,18], 
where the temperature or parameters are dynamically fluctuated with keeping the statistical descriptions.

\subsection{Reweighting}

In application, we want to have a desired distribution for a specific target system, say, $I$. This can be done using a reweighting technique, since we have a predetermined distribution described by Eqs. (32) and (34). To obtain a formula reweighting to any desired distribution density $\rho^{[I]}: \mathbb{R}^{n_{I}} \rightarrow \mathbb{R}_{+}$, we first apply Eq. (34) to function $A \rho^{[I]} \check{\rho}_{[I]} / \rho_{\mathrm{X}}$ for any quantity $A\left(x^{[I]}\right)$. Here, $\check{\rho}_{[I]}$ is a function on $\mathbb{R}^{\sum_{J=1(J \neq I)}^{N} n_{J}}$ taking a value of

$$
\check{\rho}_{[I]}\left(x^{[1]}, \ldots, x^{[I-1]}, x^{[I+1]}, \ldots, x^{[N]}\right),
$$

which is introduced to be a convergence factor, as seen below. For this role, $\check{\rho}_{[I]}$ should be strictly positive and integrable:

$0<c_{[I]} \equiv \int_{\mathbb{R}} \sum_{J=1(J \neq I)}^{N}{ }^{n} \prod_{J=1(J \neq I)}^{N} d x^{[J]} \check{\rho}_{[I]}\left(x^{[1]}, \ldots, x^{[I-1]}, x^{[I+1]}, \ldots, x^{[N]}\right)<\infty$.

Now we have

$$
\begin{aligned}
& \left\langle A \rho^{[I]} \check{\rho}_{[I]} / \rho_{\mathrm{X}}\right\rangle \\
& \equiv \int_{\Omega} A \rho^{[I]} \check{\rho}_{[I]} / \rho_{\mathrm{X}} \rho d \omega / \int_{\Omega} \rho d \omega
\end{aligned}
$$

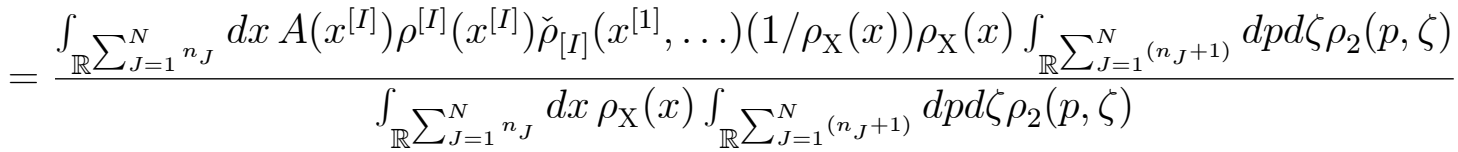

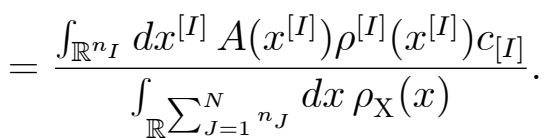

$$
\begin{aligned}
& \left\langle\rho^{[I]} \check{\rho}_{[I]} / \rho_{\mathrm{X}}\right\rangle \equiv \int_{\Omega} \rho^{[I]} \check{\rho}_{[I]} / \rho_{\mathrm{X}} \rho d \omega / \int_{\Omega} \rho d \omega \\
& =\frac{\int_{\mathbb{R}^{n_{I}}} d x^{[I]} \rho^{[I]}\left(x^{[I]}\right) c_{[I]}}{\int_{\mathbb{R} \sum_{J=1}^{N} n_{J}} d x \rho_{\mathrm{X}}(x)} .
\end{aligned}
$$

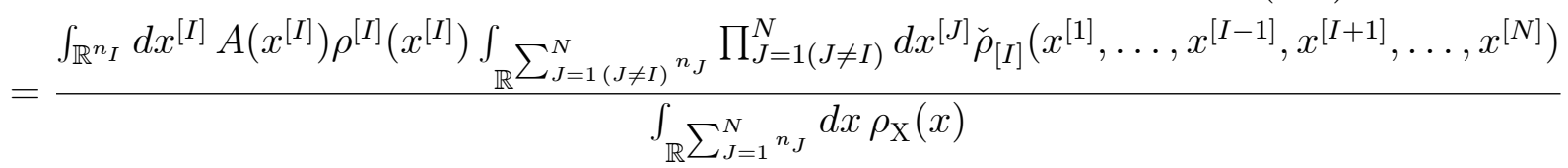


We then straightforwardly have

$$
\begin{aligned}
& \overline{A \rho \rho^{[I]} \check{\rho}_{[I]} / \rho_{\mathrm{X}}} / \overline{\rho^{[I]} \check{\rho}_{[I]} / \rho_{\mathrm{X}}} \\
& =\left\langle A \rho^{[I]} \check{\rho}_{[I]} / \rho_{\mathrm{X}}\right\rangle /\left\langle\rho^{[I]} \check{\rho}_{[I]} / \rho_{\mathrm{X}}\right\rangle \\
& =\frac{\int_{\mathbb{R}^{n_{I}}} d x^{[I]} A\left(x^{[I]}\right) \rho^{[I]}\left(x^{[I]}\right) c_{[I]}}{\int_{\mathbb{R}_{J=1} \sum_{J}} \sum^{N} \rho_{\mathrm{X}}(x)} / \frac{\int_{\mathbb{R}^{n_{I}}} d x^{[I]} \rho^{[I]}\left(x^{[I]}\right) c_{[I]}}{\int_{\mathbb{R} \sum_{J=1}^{N}{ }^{n_{J}}} d x \rho_{\mathrm{X}}(x)} \\
& =\frac{\int_{\mathbb{R}^{n_{I}}} d x^{[I]} A\left(x^{[I]}\right) \rho^{[I]}\left(x^{[I]}\right)}{\int_{\mathbb{R}^{n_{I}}} d x^{[I]} \rho^{[I]}\left(x^{[I]}\right)} \\
& =:\langle A\rangle_{\rho^{[I]}} .
\end{aligned}
$$

Thus, the average of the function of $A$ under the desired density $\rho^{[I]}$ can be obtained through the evaluations of the two long-time averages, $A \rho^{[I]} \check{\rho}_{[I]} / \rho_{\mathrm{X}}$ and $\rho^{[I]} \check{\rho}_{[I]} / \rho_{\mathrm{X}}$, via the reweighting, Eq. (44).

The factor $\check{\rho}_{[I]}$ is necessary to converge the integration with respect to $d x^{[1]} \cdots d x^{[I-1]} d x^{[I+1]} \cdots d x^{[N]}$ in Eq. (42d) (if $\check{\rho}_{[I]}=1$ then the integral becomes the infinity). Note that the reweighting formula (44) is irrelevant to the choice of specific form of $\check{\rho}_{[I]}$ in a theoretical sense. In actual simulation, however, the finite-time convergence may depend on the choice. One reasonable choice would

$\check{\rho}_{[I]}\left(x^{[1]}, \ldots, x^{[I-1]}, x^{[I+1]}, \ldots, x^{[N]}\right) \equiv \prod_{J=1(J \neq I)}^{N} \exp \left[-c^{[J]}\left\|x^{[J]}\right\|_{n_{J}}^{2}\right]$ with some positive constants $c^{[J]}(J \neq I)$.

\section{Numerics}

We numerically investigated the current method by applying it to the most basic system, a coupled system of two $1 \mathrm{HO}$ NH systems. This system is discussed in Sec. 3, and the potential function of the total system is defined by Eq. (23). The following parameter values were used: $k^{[1,1]}=k^{[2,2]}=1, k^{[1,2]}=k^{[2,1]}=$ $1 / 2, \mathrm{Q}^{[1]}=\mathrm{Q}^{[2]}=1, T^{[I]}=1, T^{[2]}=2$, and $\nu=\frac{1}{N}\left(\sum_{J=1}^{N} 1 / T^{[J]}\right)=3 / 4[$ see Eq. (20)]. For comparison, we also treat the case of $k^{[1,2]}=0$. This corresponds to a decoupled two NH EOMs (with a $1 \mathrm{HO}$ force scaled by $\nu T^{[I]}$ ).

To numerically integrating the EOMs, we used a general scheme employing an extended ODE $[19,20]$. The fundamental maps corresponding to decomposed individual vector fields are easily obtained in a similar manner described in Ref. [19], and thus a symmetric, desired order integrator can be constructed. Furthermore, the numerical error of the integration can be checked by monitoring an invariant function defined in an extended ODE during the integration process $[19,20]$. In the current study, the simplest symmetric, second-order integrator of unit time step $h=0.01$ was used. We integrated $10^{8}$ time steps with 
initial values of $x^{[1]}(0)=0, p^{[1]}(0)=1, \zeta^{[1]}(0)=0, x^{[2]}(0)=0, p^{[2]}(0)=1$, and $\zeta^{[2]}(0)=0$.

Figure 2 shows the trajectories of $\left(x^{[I]}, p^{[I]}\right)$ for the initial 50000 time steps. For the decoupled case [Figs. 2(a) and 2(b)], the trajectories show the torus-like regular structure as seen in many literature. In contrast, for the coupled case [Figs. 2(c) and 2(d)], the trajectories show chaotic behavior and explored much more compared with the decoupled case. The system with the hot thermostat $[I=2$, shown in Fig. 2(d)] sampled wider range of momentum than the system with the cold thermostat $[I=1$, shown in Fig. 2(c)].

As demonstrated, due to the scaled force, the current coupled system allows the description of the equilibrium states. Namely, this system has an invariant measure $\rho d \omega$, where $\rho$ is given by Eq. (8). Furthermore, the current simple potential function, Eq. (23), yields the explicit form of the distribution of coordinate $x^{[I]}$. That is, from Eq. (39), we have

$$
\begin{aligned}
\rho_{x^{[1]}}(y) & =\int_{\mathbb{R}^{1}} d x^{[2]} \rho_{\mathrm{X}}\left(y, x^{[2]}\right) / \int_{\mathbb{R}^{2}} d x \rho_{\mathrm{X}}(x) . \\
& \propto \int_{\mathbb{R}^{1}} d x^{[2]} \exp \left[-\nu U\left(y, x^{[2]}\right)\right] \\
& \propto \exp \left[-\frac{\nu}{2}\left\{k^{[1,1]}-\frac{\left(k^{[1,2]}\right)^{2}}{k^{[2,2]}}\right\} y^{2}\right] .
\end{aligned}
$$

Similarly we have

$$
\rho_{x^{[2]}}(y) \propto \exp \left[-\frac{\nu}{2}\left\{k^{[2,2]}-\frac{\left(k^{[1,2]}\right)^{2}}{k^{[1,1]}}\right\} y^{2}\right] .
$$

In the current numerics we thus have $\rho_{x^{[1]}}(y)=\rho_{x^{[2]}}(y)=\sqrt{\frac{9}{32 \pi}} \exp \left[-\frac{9}{32} y^{2}\right]$. The distribution density of $p^{[I]}$ and that of $\zeta^{[I]}$ are always Gaussian function [see Eq. (8)], and the current case yields: $\rho_{p^{[1]}}(y)=\rho_{\zeta^{[1]}}(y)=\frac{1}{\sqrt{2 \pi}} \exp \left[-\frac{1}{2} y^{2}\right]$ and $\rho_{p^{[2]}}(y)=\rho_{\zeta^{[2]}}(y)=\frac{1}{2 \sqrt{\pi}} \exp \left[-\frac{1}{4} y^{2}\right]$.

We confirm that these distributions theoretically obtained agree with the distributions numerically produced. The latter were obtained by the histogram counted in the numerical simulation, viz., by the long-time average [Eq. (17)] $\overline{\chi_{C}} /|C|$ (with a finite-time approximation), where $\chi_{C}$ is the characterized function with respect to each small bin $C$ for an individual variable. Figure 3 shows these theoretical and simulated distribution densities. Simulated distribution densities show clean-cut Gaussian shapes and the agreement with the theoretical densities are good for all the variables. This indicates that $\overline{\chi_{C}}=\left\langle\chi_{C}\right\rangle$ for all bins and for all variables. Thus, the invariant density, or stationary density, $\rho$, given by Eq. (8), was realized under the ergodic condition in the current system. In this sense, the current coupling method produces an equilibrium in the system that is composed from the NH system with a hot thermostat and 
the NH system with a cold thermostat, and the method allows a completely definite statistical description. Although the ergodicity is broken in the single $\mathrm{NH} 1 \mathrm{HO}$ system (viz., the decoupled case in the current study) $[2,21]$ and identical 1HOs coupled to the single NH equation [22], it holds in the current coupled NH $1 \mathrm{HO}$ systems. Only the current coupling with the additional linear force $-\nu T^{[I]} k^{[I, J]} x^{[J]}$ against particle $I$ is sufficient to attain the ergodicity.

These are the conclusions obtained from the numerical simulations. Pure mathematical statement related to ergodic dynamical system theory is still open. Applications to more complicated systems are under study.

Acknowledgments The author thanks Kei Moritsugu for useful discussions. This work was partially supported by a Grant-in-Aid for Scientific Research (C) (25390156) and by the "Development of core technologies for innovative drug development based upon IT" from Japan Agency for Medical Research and development, AMED.

\section{References}

[1] S. Nosé, J. Chem. Phys. 81, 511 (1984).

[2] W.G. Hoover, Phys. Rev. A 31, 1695 (1985).

[3] S. Nosé, Prog. Theor. Phys. Suppl. 103, 1 (1991).

[4] W. G. Hoover, Computational Statistical Mechanics (Elsevier, N.Y., 1991).

[5] W. G. Hoover, Time Reversibility, Computer Simulation, Algorithms, Chaos (World Scientific, 2012).

[6] K. Aoki and D. Kusnezov, Phys. Rev. Lett. 86, 4029 (2001).

[7] W.G. Hoover and C.G. Hoover, Comm. Nonlinear Sci. Numer. Sim. 18, 3365 (2013).

[8] K. Kaneko, Prog. Theor. Phys. 74, 1033 (1985).

[9] M. Cross and P. Hohenberg, Rev. Mod. Phys. 65, 851 (1993).

[10] Y. Sugita and Y. Okamoto, Chem. Phys. Lett. 314, 141(1999).

[11] I. Fukuda and H. Nakamura, Phys. Rev. E 65, 026105 (2002).

[12] I. Fukuda, J. Chem. Phys. 132, 127101 (2010).

[13] D. J. Tobias, G. J. Martyna, and M. L. Klein, J. Phys. Chem. 97, 12959 (1993).

[14] P. H. Hünenberger, Advanced Computer Simulation: Approaches for Soft Matter Sciences I. (Advances in Polymer Science vol 173) eds. C. Holm and K. Kremer, 105-49 (Springer, Berlin, 2005). 
[15] G. J. Martyna, M. L. Klein, and M. Tuckerman, J. Chem. Phys. 97, 2635 (1992).

[16] W. G. Hoover and B. L. Holian, Phys. Lett. A 211, 253 (1996).

[17] I. Fukuda and K. Moritsugu, J. Phys. A 48, 455001 (2015).

[18] I. Fukuda and K. Moritsugu, Phys. Rev. E (2016) In press.

[19] I. Fukuda and H. Nakamura, Phys. Rev. E 73, 026703 (2006).

[20] S. Queyroy, H. Nakamura, and I. Fukuda, J. Comp. Chem. 30, 1799 (2009).

[21] H. A. Posch, W. G. Hoover, and F. J. Vesely, Phys. Rev. A 33, 4253 (1986).

[22] S. Nosé, Phys. Rev. E 47, 164 (1993). 

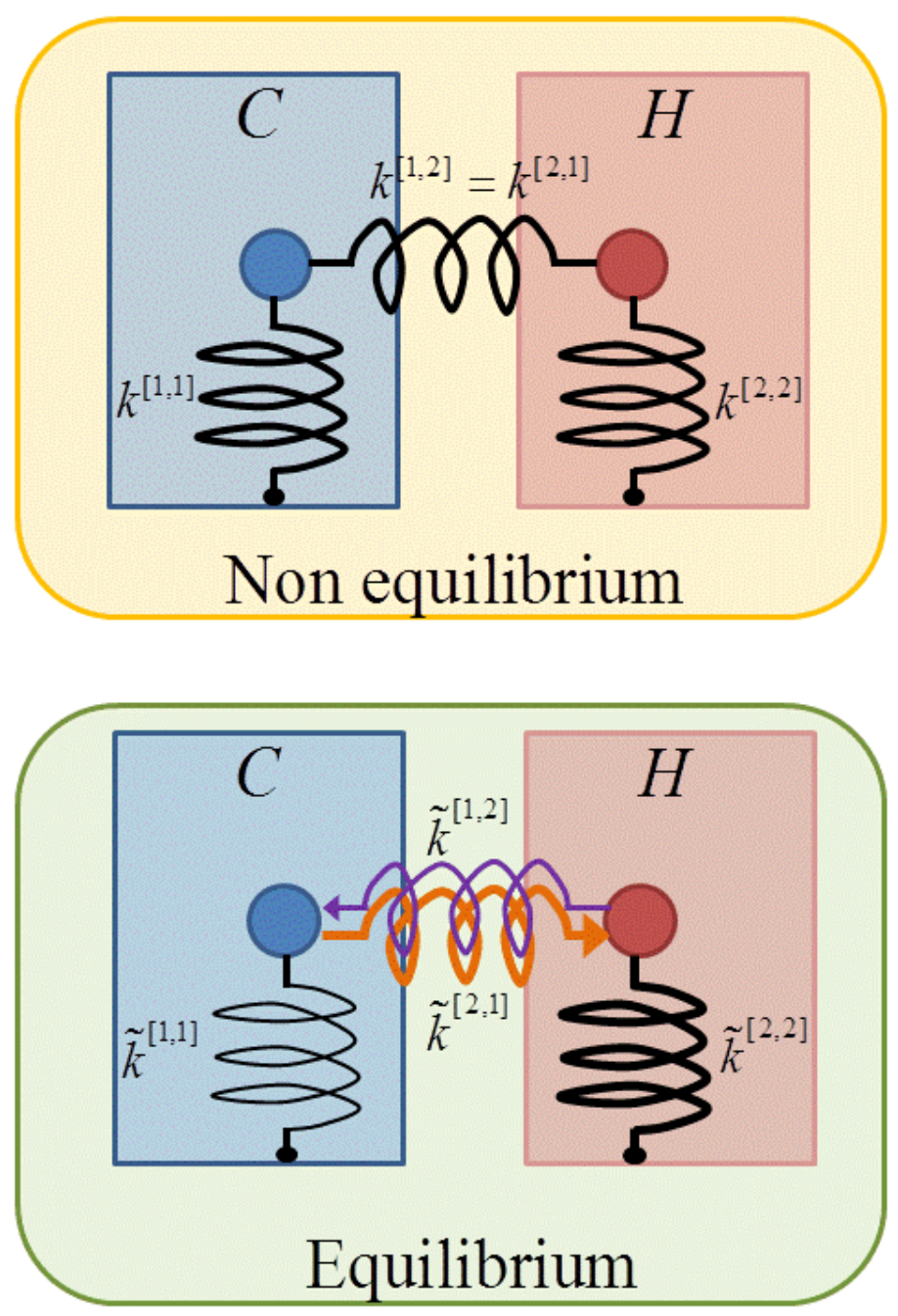

Fig. 1. Schematic figure to understand the current scheme by presenting the most simple example of the coupled NH lattices. Two oscillators are subject to cold and hot bath, respectively. They also have an interaction described by a spring with its strength $k^{[1,2]}$. (a) Conventional case represents a non equilibrium. (b) The current case realizes an equilibrium by using scaled forces, viz., the scaled spring constants, $\tilde{k}^{[I, J]}=\nu T^{[I]} k^{[I, J]}$, which is not symmetric in general [see Eq. (24)]. 

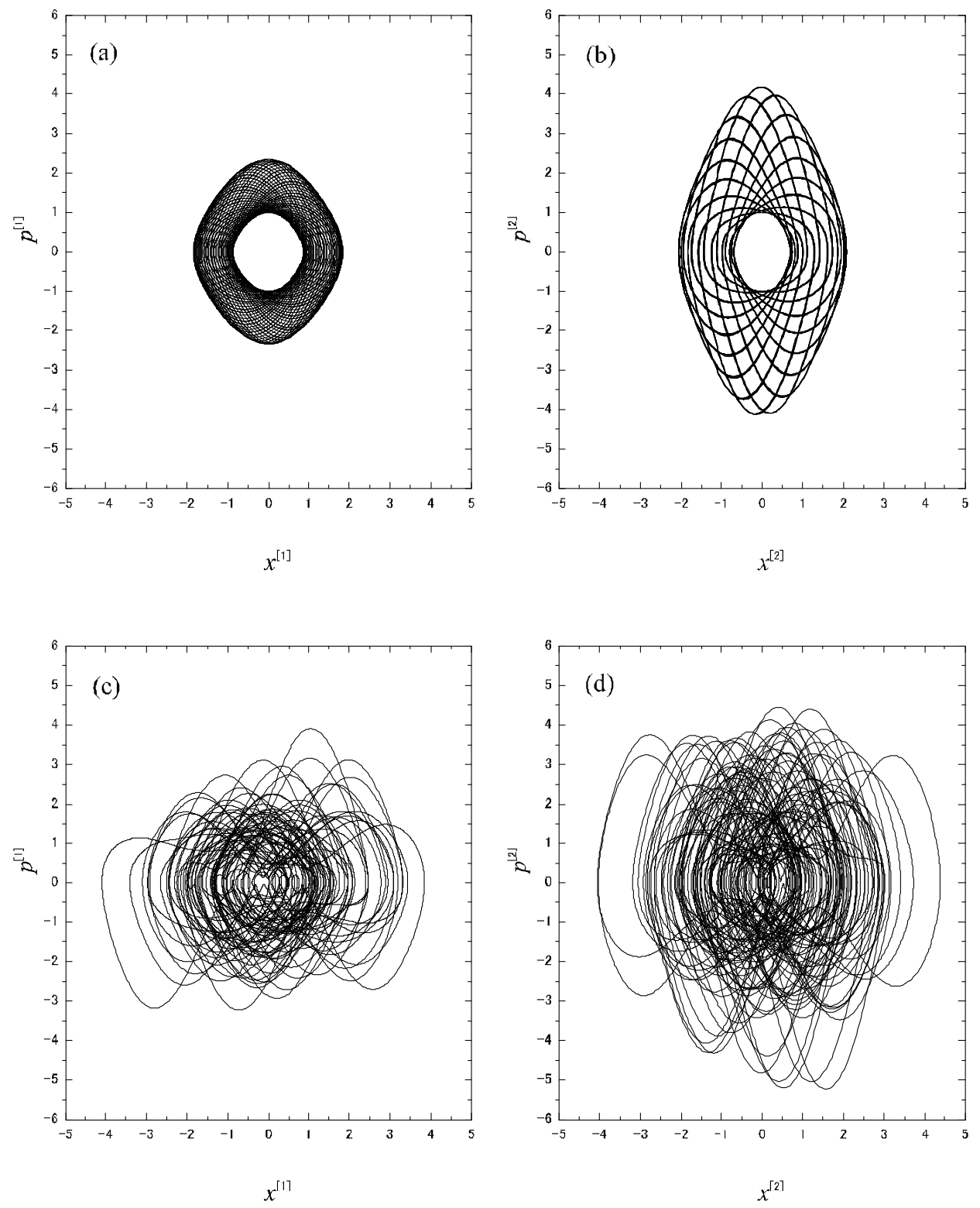

Fig. 2. $(x, p)$ trajectories for $\mathrm{NH}$ oscillators: (a) an oscillator in a cold bath in an uncoupled system, (b) an oscillator in a hot bath in an uncoupled system, (c) an oscillator in a cold bath in the coupled system, and (d) an oscillator in a hot bath in the coupled system. 

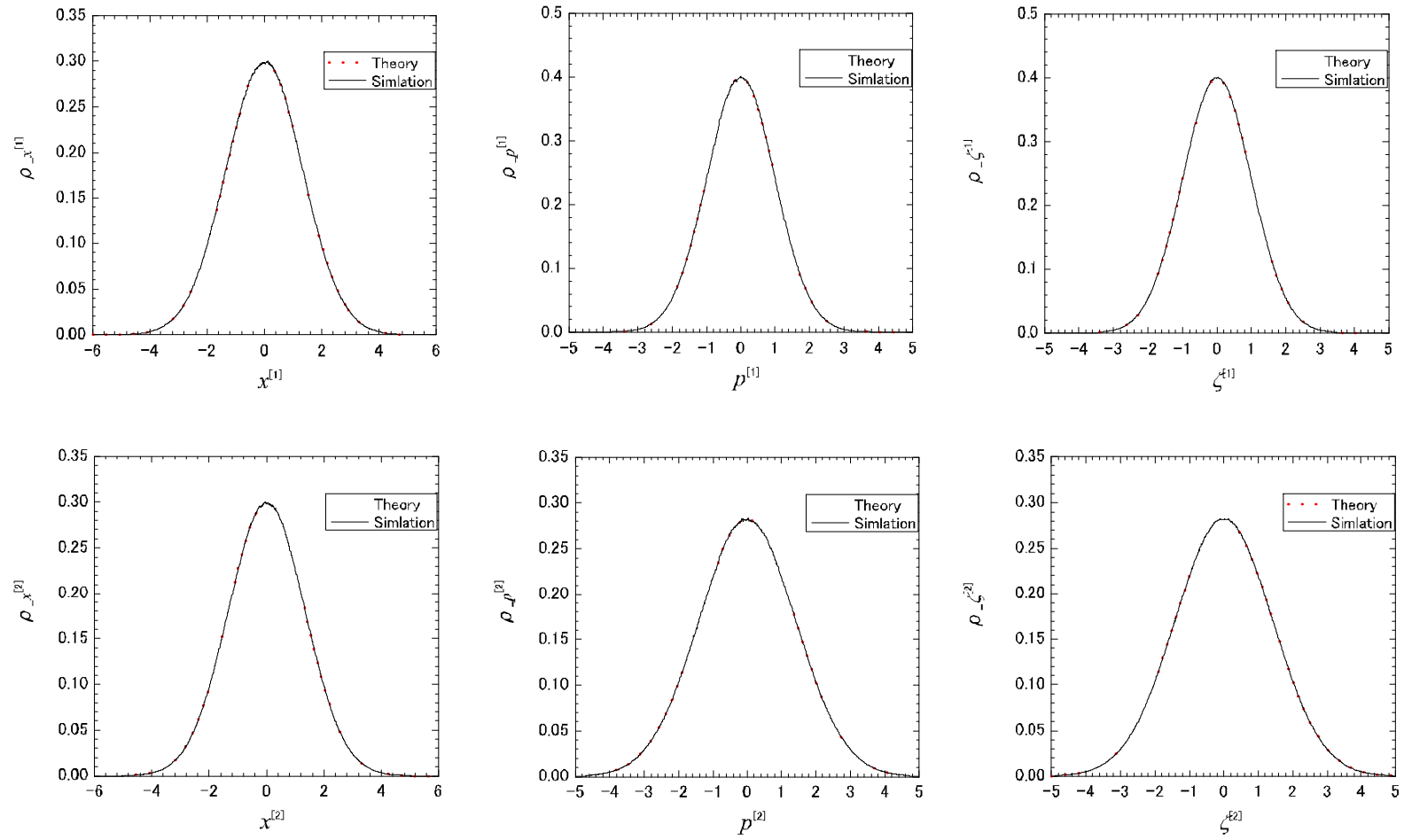

Fig. 3. Distribution densities for the coupled NH oscillators: (top) theoretical and simulated densities of $x, p, \zeta$ for system 1 (an oscillator in a cold bath); (bottom) theoretical and simulated densities of $x, p, \zeta$ for system 2 (an oscillator in a hot bath). 